\title{
Successful Treatment of Severe Newly Diagnosed Immune Thrombocytopenia Involving an Alveolar Hemorrhage with Combination Therapy Consisting of Romiplostim, Rituximab and Vincristine
}

\author{
Kiyoshi Okazuka ${ }^{1}$, Masayoshi Masuko ${ }^{2}$, Yuji Matsuo ${ }^{1}$, Shukuko Miyakoshi ${ }^{1}$, \\ Tomoyuki Tanaka ${ }^{1}$, Takashi Kozakai ${ }^{1}$, Hironori Kobayashi ${ }^{1}$, Kyoko Fuse ${ }^{1}$, \\ Yasuhiko Shibasaki ${ }^{1}$, Masato Moriyama ${ }^{1}$, Jun Takizawa ${ }^{1}$, Ichiro Fuse ${ }^{3}$, \\ Ken Toba ${ }^{1}$ and Tatsuo Furukawa ${ }^{2}$
}

\begin{abstract}
A 51-year-old man was admitted due to a severe bleeding tendency. After he was diagnosed with immune thrombocytopenia (ITP), several therapies, including steroids, steroid pulse, vincristine and rituximab, were administered; however, the patient's bleeding symptoms were not sufficiently controllable with these treatments. Subsequently, a diffuse alveolar hemorrhage was observed. Treatment with a thrombopoietin receptor agonist, romiplostim, was initiated to prevent lethal hemorrhaging, although the efficacy of thrombopoietic receptor agonists in such emergency situations has not been elucidated. The initiation of romiplostim achieved prompt remission in platelets. This case suggests that combination therapy with romiplostim, rituximab and vincristine is effective in cases of newly diagnosed severe therapy-resistant ITP.
\end{abstract}

Key words: ITP, thrombopoietin receptor agonist, romiplostim, diffuse alveolar hemorrhage

(Intern Med 52: 1239-1242, 2013)

(DOI: 10.2169/internalmedicine.52.0080)

\section{Introduction}

Immune thrombocytopenia (ITP) is an acquired disease characterized by isolated thrombocytopenia involving a platelet count of less than $100 \times 10^{3} / \mu \mathrm{L}$. Thrombocytopenia is caused by platelet destruction via immune-mediated mechanisms and inadequate platelet production. Adult ITP patients usually display a chronic course, and the most common manifestation is mucocutaneous or subcutaneous bleeding, including petechiae, purpura and epistaxis. Lethal hemorrhaging is a rare complication of ITP, and thrombocytopenia involving a platelet count of less than $30 \times 10^{3} / \mu \mathrm{L}$ carries a 4.2-fold increased mortality risk compared with thrombocytopenia involving a platelet count of more than $30 \times 10^{3} /$ $\mu \mathrm{L}$ (1). In particular, patients with very severe thrombocy- topenia involving a platelet count of less than $10 \times 10^{3} / \mu \mathrm{L}$ must be treated immediately to prevent life-threatening bleeding.

When it is necessary to rapidly increase a patient's platelet count due to the presence of life-threatening bleeding, such as intracranial bleeding or severe organ bleeding, the use of intravenous immunoglobulin (IVIg) or corticosteroid pulse therapy is recommended (2). Platelet transfusions are also used in the emergency setting; however, their efficacy is usually transient. Furthermore, $10-20 \%$ of patients are refractory to initial treatment with corticosteroids and/or IVIg therapy. The American Society of Hematology proposed evidence-based practice guidelines for ITP, in which splenectomy is recommended for patients who are unresponsive to initial therapy (3). Although splenectomy is a curative treatment, a higher perioperative morbidity of this procedure

${ }^{1}$ Division of Hematology, Niigata Univerisity Medical and Dental Hospital, Japan, ${ }^{2}$ Division of Bone Marrow Transplantation, Niigata University Medical and Dental Hospital, Japan and ${ }^{3}$ Department of Transfusion Medicine, Niigata University Medical and Dental Hospital, Japan Received for publication January 9, 2013; Accepted for publication February 15, 2013 Correspondence to Dr. Kiyoshi Okazuka, kiyo.okazuka@gmail.com 


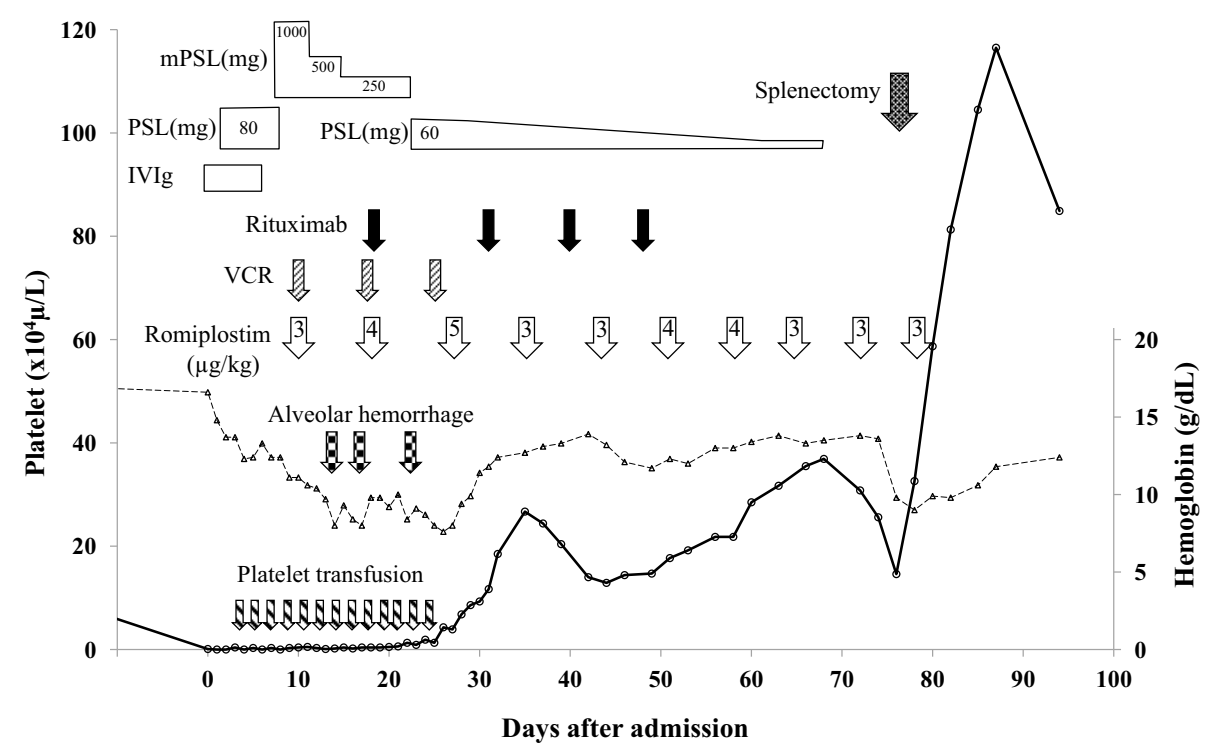

Figure 1. The clinical course of the patient's platelet count and the therapies employed against ITP. The $\mathrm{X}$-axis represents the day after hospitalization, and the $\mathrm{Y}$-axis represents the platelet count and hemoglobin level in the peripheral blood. Solid and dashed lines represent the number of platelet and the level of hemoglobin, respectively.

has been reported among patients with severe thrombocytopenia compared with those with higher platelet counts (4).

The newly developed thrombopoietin (TPO) receptor agonist romiplostim has been reported to display high efficacy with tolerable adverse effects against refractory chronic ITP $(5,6)$. However, the optimal second-line therapy for newly diagnosed patients in whom corticosteroid therapy fails remains disputed. Thus far, there have been only a few case reports of the early management of newly diagnosed severe ITP using romiplostim after the failure of conventional therapy (7). Therefore, the efficacy and safety of romiplostim against severe newly diagnosed ITP have not been fully elucidated. We herein report a case of newly diagnosed severe ITP that was treated with romiplostim after the failure of several initial treatments.

\section{Case Report}

A 51-year-old man with dyslipidemia and depression was treated with paroxetine hydrochloride hydrate and rosuvastatin at our hospital. His laboratory data were checked periodically, and he displayed a normal platelet count of $141 \times$ $10^{3} / \mu \mathrm{L} 28$ days prior to admission. However, petechiae and mucosal bleeding in his mouth suddenly developed two days before his next consultation. At that point, his platelet count was less than $1.0 \times 10^{3} / \mu \mathrm{L}$; however, no findings indicative of disseminated intravascular coagulation (DIC) were detected. In addition, the patient had not suffered from any obvious infection episodes prior to developing thrombocytopenia, nor had he recently started taking any medications. Therefore, we diagnosed him with ITP and administered $400 \mathrm{mg} / \mathrm{kg}$ of immunoglobulin (IVIg) for five days. Since no increases in the platelet count were obtained by two days after the start of the IVIg treatment and the patient's bleeding tendency gradually worsened, e.g., he displayed macrohematuria, a bone marrow aspiration was performed to confirm his diagnosis. The bone marrow examination revealed an increased level of megakaryocytes but no findings that were indicative of any other disease that could cause thrombocytopenia. In addition, tests for Helicobacter pylori, hepatitis $\mathrm{C}$ virus and human immunodeficiency virus were negative. Therefore, treatment with $1 \mathrm{~g}$ of methylprednisolone (mPSL) was administered for three days and then gradually tapered. Until nine days after admission, the patient's platelet count did not show any increases, fluctuating between $1.0 \times 10^{3}$ and $3.0 \times 10^{3} / \mu \mathrm{L}$, despite treatment with IVIg, mPSL and 60 to 80 units of platelet transfusion. Severe thrombocytopenia unresponsive to these treatments resulted in worsening macrohematuria and oozing of blood from the site of the bone marrow aspiration. To prevent lethal hemorrhaging, such as a brain hemorrhage, due to severe thrombocytopenia, weekly treatment with $2 \mathrm{mg}$ of vincristine, an off-label drug in Japan, was initiated starting on day 9 with the expectation of rapidly improving the platelet count. Since the time to an initial response to vincristine is usually between seven and 14 days, a thrombopoietin receptor agonist, romiplostim, was administered to prevent lethal hemorrhaging, although the efficacy of thrombopoietic receptor agonists in such emergency situations has not so far been elucidated. Since no severe adverse events were observed in a phase II study of 3 $\mu \mathrm{g} / \mathrm{kg}$ of romiplostim (8), we initiated treatment with $3 \mu \mathrm{g} /$ $\mathrm{kg}$ of romiplostim starting on day 12 for immediate recovery of the platelet count. Because severe thrombocytopenia remained despite the administration of several treatments, the patient developed severe hypoxia due to a diffuse alveolar hemorrhage (DAD) on day 14 (Fig. 2). Even a platelet 


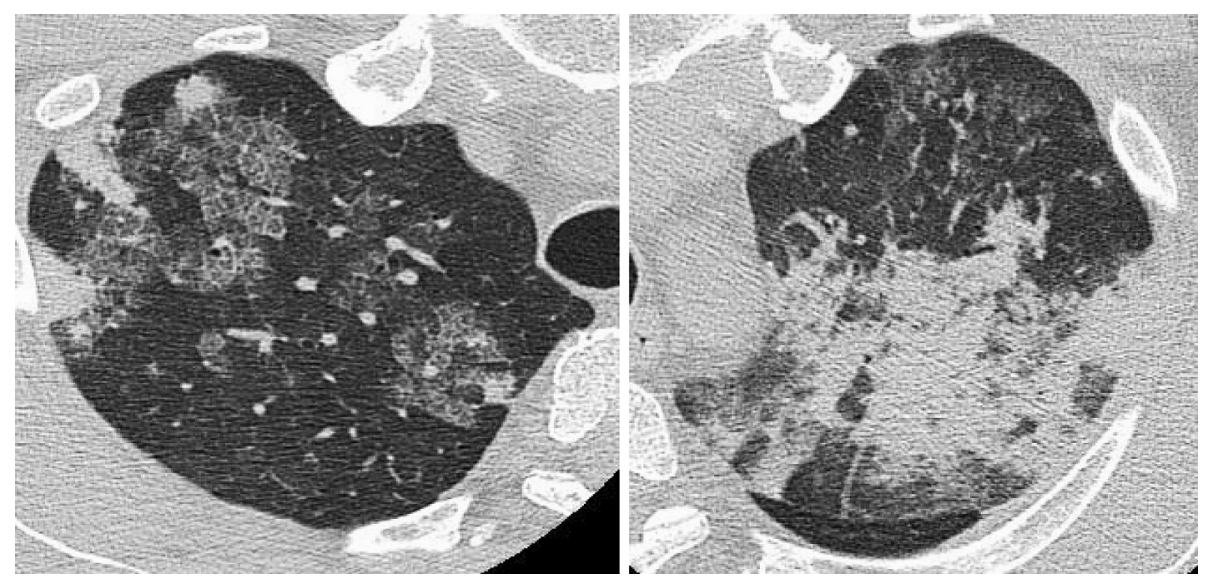

Figure 2. A CT scan showing the diffuse alveolar hemorrhage observed on day 14 . The right and left photos show the right and left upper lobes, respectively.

transfusion failed to achieve any increases in the platelet count; therefore, treatment with recombinant factor VIIa, which is also an off-label drug for ITP in Japan, was initiated to stop the DAD with the use of noninvasive positive pressure ventilation (9). While the DAD gradually improved following the initiation of these treatments, it occurred again on day 17 because the platelet count did not improve thoroughly; thus, severe progressive anemia was observed. Therefore, $375 \mathrm{mg} / \mathrm{m}^{2}$ of rituximab was administered on day 19 in consideration of the failure of the other treatments. On day 18 , the dose of romiplostim was increased from 3 to 4 $\mu \mathrm{g} / \mathrm{kg}$, and the patient's platelet count finally increased to $6 \times$ $10^{3} / \mu \mathrm{L}$ and $13 \times 10^{3} / \mu \mathrm{L}$ on days 21 and 22 , respectively. In addition, the diffuse alveolar hemorrhage gradually improved in accordance with the increase in the platelet count.

The administration of romiplostim was continued until the patient underwent splenectomy in order to maintain his platelet count. Splenectomy was performed successfully on day 74 because the patient did not wish to keep taking romiplostim, thus resulting in the recovery of his platelet count and the discontinuation of all treatments for ITP.

\section{Discussion}

We herein report that the administration of romiplostim improved the platelet count of a patient with newly diagnosed severe ITP involving life-threatening hemorrhagic symptoms that were unresponsive to corticosteroids and IVIg. Although IVIg and corticosteroids are generally effective against severe ITP in the emergency setting, approximately $20-30 \%$ of ITP patients are unresponsive to treatment with these therapies (10). Second-line treatment is recommended in cases of resistance to first-line treatments. However, the time to a response after the initiation of a secondline treatment is usually longer than that observed after the first-line treatment. In patients with severe ITP, their platelet count must be immediately increased above $30 \times 10^{9} / \mathrm{L}$ to prevent lethal bleeding symptoms since thrombocytopenia involving a platelet count of less than $30 \times 10^{9} / \mathrm{L}$ is associated with a 4.2-fold increased mortality risk compared with thrombocytopenia involving a platelet count of more than $30 \times 10^{9} / \mathrm{L}$ (1). Therefore, especially in patients with severe ITP, treatment strategies that can rapidly achieve a durable remission are crucial.

According to the treatment guidelines for ITP published by the American Society of Hematology, the initiation of anti-D antibody treatment is recommended as an initial treatment (3) because the time to response after this treatment is generally short; i.e., 1-14 days (11). However, anti$\mathrm{D}$ antibodies are not available in many countries due to safety concerns. Recently, many reports have described the efficacy of rituximab against ITP (12). While rituximab displays significant efficacy against ITP, even refractory or resistant ITP, it is also associated with a longer time to response compared with first-line treatments. Therefore, rituximab may be an alternative treatment for resistant chronic ITP, but not for severe ITP involving severe bleeding symptoms. Furthermore, splenectomy is expected to achieve a durable response, even in resistant patients; however, the safety of splenectomy in patients with severe bleeding tendencies is open to question. Therefore, severe ITP patients with lethal bleeding tendencies in whom first-line treatment fails must receive other treatments to achieve rapid platelet recovery.

Since thrombopoietin receptor agonists have been licensed, it has been reported that the time to response after the administration of romiplostim is shorter than that observed after treatment with other second-line modalities (13); i.e., less than 14 days with minor adverse events. It has also been clearly demonstrated that thrombopoietin receptor agonists are effective and safe against persistent or chronic ITP $(6,14)$. However, the efficacy of romiplostim has not been elucidated in patients with severe newly diagnosed ITP, especially those with severe bleeding symptoms. We administered romiplostim to a newly diagnosed severe ITP patient following the failure of IVIg and PSL therapy, which resulted in a rapid increase in the patient's platelet count. In addition, the response rate of rituximab is markedly lower than that of other treatments (13). Therefore, given its high response rate, romiplostim can be considered 
to be the optimal second-line treatment for achieving rapid platelet recovery, even in newly diagnosed severe ITP patients.

In our case, platelet recovery was obtained soon after the administration of VCR, rituximab and romiplostim. Although the time to initial response in the platelet count following the initiation of rituximab is usually longer than that for romiplostim, some patients show a response seven days after the start of treatment. In addition, the time to initial response to VCR is usually between seven and 14 days (15), which is almost identical to the time required for platelet recovery in our case. Therefore, in our case, combination therapy consisting of VCR, rituximab and romiplostim achieved platelet recovery after failure of mPSL and IVIg therapy in a patient with severe newly diagnosed ITP.

While it has not been clarified as to whether splenectomy is necessary after platelet remission has been achieved with romiplostim, this procedure remains the most effective therapy for resistant ITP. Since the risk of severe intraoperative bleeding is high, preoperative ITP treatment should achieve a preoperative platelet count of greater than $50 \times 10^{3} / \mu \mathrm{L}$ (16). Although the administration of romiplostim before splenectomy is also an off-label use in Japan, romiplostim may be a suitable treatment for increasing a patient's platelet count before splenectomy in order to prevent lethal bleeding complications. While there is no evidence regarding the timing of splenectomy in adult patients with ITP, Provan et al. recommended that splenectomy be deferred for at least six months after diagnosis (13). Our patient strongly hoped to stop taking romiplostim after achieving platelet remission; therefore, we performed splenectomy after obtaining informed consent regarding the risk of splenectomy, including infection.

In conclusion, our case demonstrated the efficacy of combination therapy consisting of romiplostim, rituximab and vincristine against newly diagnosed severe ITP that displayed resistance to conventional initial treatment. Given the rapidity of the response and the fact that the drug was associated with few side effects, romiplostim should be considered as a second-line treatment in patients with severe newly diagnosed ITP showing severe lethal bleeding. Although it has been reported that romiplostim achieves a significant response without severe adverse events in most cases, it has also been reported that some patients exhibit reticlin deposition, thrombosis or the development of leukemia following the administration of romiplostim $(17,18)$. Since only two years of postmarketing data are available in which no severe adverse events were documented (6), additional long-term follow-up of patients is necessary to draw any definitive conclusions regarding the safety of romiplostim. Therefore, other treatment modalities must be initiated before romiplostim, and romiplostim should be administered with careful observation
The authors state that they have no Conflict of Interest (COI).

\section{References}

1. Portielje JE, Westendorp RG, Kluin-Nelemans HC, Brand A. Morbidity and mortality in adults with idiopathic thrombocytopenic purpura. Blood 97: 2549-2554, 2001.

2. Provan D, Newland AC. Guidelines for immune thrombocytopenia. N Engl J Med 364: 580-581-authorreply581, 2011.

3. Neunert C, Lim W, Crowther M, Cohen A, Solberg L, Crowther MA. The American Society of Hematology 2011 evidence-based practice guideline for immune thrombocytopenia. Blood 117: 4190-4207, 2011.

4. Keidar A, Sagi B, Szold A. Laparoscopic splenectomy for immune thrombocytopenic purpura in patients with severe refractory thrombocytopenia. Pathophysiol Haemost Thromb 33: 116-119, 2003.

5. Bussel JB, Buchanan GR, Nugent DJ, et al. A randomized, double-blind study of romiplostim to determine its safety and efficacy in children with immune thrombocytopenia. Blood 118: 2836, 2011.

6. Khellaf M, Michel M, Quittet P, et al. Romiplostim safety and efficacy for immune thrombocytopenia in clinical practice: 2-year results of 72 adults in a romiplostim compassionate-use program. Blood 118: 4338-4345, 2011.

7. Tagariello G, Sartori R, Radossi P, Maschio N, Risato R, Stasi R. Romiplostim for the early management of severe immune thrombocytopenia unresponsive to conventional treatment. Br J Haematol 157: 256-258, 2012.

8. Shirasugi Y, Ando K, Hashino S, et al. A phase II, open-label, sequential-cohort, dose-escalation study of romiplostim in Japanese patients with chronic immune thrombocytopenic purpura. Int J Hematol 90: 157-165, 2009.

9. Hicks K, Peng D, Gajewski JL. Treatment of diffuse alveolar hemorrhage after allogeneic bone marrow transplant with recombinant factor VIIa. Bone Marrow Transplant 30: 975-978, 2002.

10. Vianelli N, Valdrè L, Fiacchini M, et al. Long-term follow-up of idiopathic thrombocytopenic purpura in 310 patients. Haematologica 86: 504-509, 2001.

11. Rodeghiero F, Stasi R, Gernsheimer T, et al. Standardization of terminology, definitions and outcome criteria in immune thrombocytopenic purpura of adults and children: report from an international working group. Blood 113: 2386-2393, 2009.

12. Stasi R, Pagano A, Stipa E, Amadori S. Rituximab chimeric antiCD20 monoclonal antibody treatment for adults with chronic idiopathic thrombocytopenic purpura. Blood 98: 952-957, 2001.

13. Provan D, Stasi R, Newland AC, et al. International consensus report on the investigation and management of primary immune thrombocytopenia. Blood 115: 168-186, 2010.

14. Kuter D, Rummel M, Boccia R, et al. Romiplostim or standard of care in patients with immune thrombocytopenia. $\mathrm{N}$ Engl $\mathrm{J}$ Med 363: 1-11, 2010.

15. George JN, Woolf SH, Raskob GE, et al. Idiopathic thrombocytopenic purpura: a practice guideline developed by explicit methods for the American Society of Hematology. Blood 88: 3-40, 1996.

16. Habermalz B, Sauerland S, Decker G, et al. Laparoscopic splenectomy: the clinical practice guidelines of the European Association for Endoscopic Surgery (EAES). Surg Endosc 22: 821-848, 2008.

17. Gardner K, Mathe S, Sahu S. Leukaemic transformation with romiplostim. Br J Haematol 158: 153, 2012.

18. Kuter DJ, Mufti GJ, Bain BJ, Hasserjian RP, Davis W, Rutstein M. Evaluation of bone marrow reticulin formation in chronic immune thrombocytopenia patients treated with romiplostim. Blood 114: 3748-3756, 2009

(C) 2013 The Japanese Society of Internal Medicine http://www.naika.or.jp/imonline/index.html 